\title{
A Female Patient with FMR1 Premutation and Mosaic X Chromosome Aneuploidy and Two Sons with Intellectual Disability
}

\author{
Ekaterina M. Galaninaa, c Andrey A. Tulupovb,c Natalya A. Lemskaya ${ }^{a}$ \\ Aleksandra M. Korostyshevskaya ${ }^{b}$ Yuliya V. Maksimovad, e Asia R. Shorina ${ }^{e}$ \\ Andrey A. Savelov ${ }^{b}$ Irina G. Sergeeva ${ }^{c}$ Evgeniya R. Isanovac \\ Irina V. Grishchenko ${ }^{a, c}$ Dmitry V. Yudkin ${ }^{\text {a, c }}$ \\ ${ }^{a}$ Chromosome Pathology Group, Institute of Molecular and Cellular Biology, and ${ }^{b}$ International Tomography \\ Center, SB RAS, 'Department of Medicine, Novosibirsk State University, dNovosibirsk State Medical University, and \\ eNovosibirsk State Regional Clinical Diagnostic Center, Novosibirsk, Russia
}

\section{Established Facts}

- Fragile X syndrome is the main cause of inherited intellectual disability in human.

- Female carriers transmit the syndrome to their sons.

\section{Novel Insights}

- The female carrier described here has supernumerary X chromosomes and X-derived marker chromosomes and 2 sons with intellectual disability of different nature.

\section{Keywords}

Aneuploidy $\cdot F M R 1 \cdot$ Fragile $X$ syndrome $\cdot$ Intellectual disability $\cdot X$ chromosome her sons have ID and a normal chromosome number. One of the sons has fragile $X$ syndrome, and the other has ID of an unclear nature.

\begin{abstract}
In this report, we describe a molecular cytogenetic study of a family burdened with intellectual disability (ID) and suicide. Our study revealed that the mother has a heterozygous premutation in the FMR1 gene and supernumerary $\mathrm{X}$ chromosomes as well as $\mathrm{X}$-derived marker chromosomes. Both of
\end{abstract}

Intellectual disability (ID) is very widespread in the human world population, being observed at a rate of approximately $1 \%$. The nature of ID is extremely heterogeneous and can have genetic or environmental reasons. Genetic causes of ID are also extremely different and can 
be connected with genes on either autosomes or sex chromosomes. Currently, there are more than 700 genes that can cause ID, and this number is increasing [Vissers et al., 2016].

Fragile X syndrome (FXS) is the main reason for inherited ID in humans. This syndrome develops due to the expansion of CGG repeats in the $5^{\prime}$ UTR of the FMR1 gene in the $\mathrm{X}$ chromosome. The normal allele contains no more than 55 repeats; the fully mutated allele contains more than 200 repeats [D'Hulst and Kooy, 2009]. This syndrome is characterized by different degrees of ID. Women heterozygous for FMR1 alleles tend to lack $1 \mathrm{X}$ chromosome and have a high risk for the development of mosaic Turner syndrome [Dobkin et al., 2009].

Sex chromosome number abnormalities are frequent and are found at a rate of approximately 1/500 in men and $1 / 2,000$ in women [Griffiths et al., 1999]. Supernumerary $\mathrm{X}$ chromosomes do not usually have any effect on carriers, but some abnormalities can arise. Twenty-five percent of triple-X women had ID, and a tendency to an increasing incidence is observed in tetra- and pentasomy $\mathrm{X}$ cases [Fryns et al., 1983]. Supernumerary sex chromosomes can sometimes influence language development [Lee et al., 2012].

In this study, we report a clinical and molecular cytogenetic study of a family that has ID based on FXS in one son and ID of an unclear nature in the other son. Their mother has supernumerary $\mathrm{X}$ chromosomes and X-derived marker chromosomes.

\section{Materials and Methods}

\section{Clinical and MRI Study}

The clinical study included consultations with medical specialists such as clinical psychologists, neurologists, and speech therapists. MRI scanning was performed with a 1.5-T whole-body MRIscanner (Philips, Achieva). The 44-year-old woman and her 20 -year-old son were examined with noncontrast routine MRI including T1- and T2-weighted imaging, FLAIR, DWI, and myelography sequences.

\section{Cell Culture and Metaphase Preparation}

B lymphocytes from each patient were obtained from peripheral blood. Cells were cultivated in RPMI 1640 GlutaMAX medium (Gibco) with 15\% fetal bovine serum (Gibco) and antibiotics. Metaphases were prepared in Carnoy's fixative as previously described [Yudkin et al., 2014]. G-banding was performed according to the standard method.

\section{Fluorescence in situ Hybridization}

BAC clones containing the FMR1 gene (RP11-489K19) and the GPR50 gene (RP11-351H6) (Empire Genomics) were labeled with a NICK translation kit (Invitrogen) with biotin or digoxigenin.

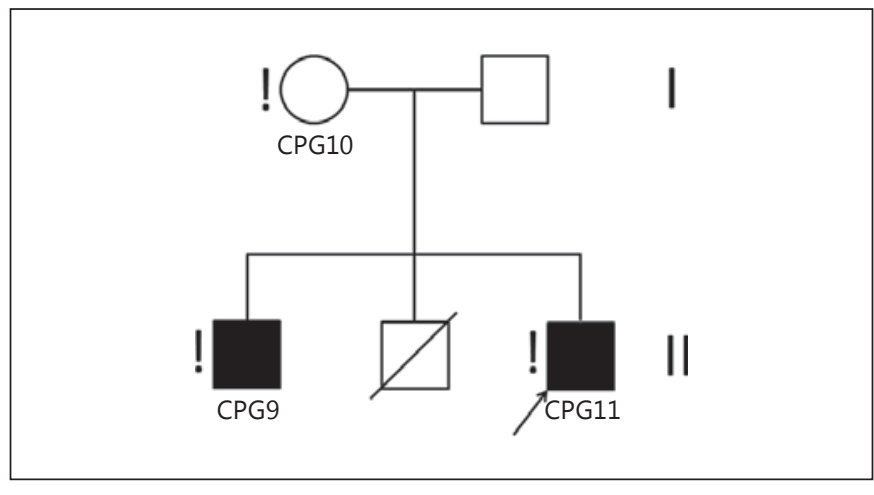

Fig. 1. Pedigree of the family. The arrow indicates the proband. The exclamation marks indicate the affected individuals.

The X chromosome painting probe was labeled with biotin or digoxigenin in DOP-PCR with a 6MW primer [Telenius et al., 1992]. GPR50 is a more telomeric sequence than FMR1 and helps to analyze the results because the fragile site FRAXA is located close to the telomere and difficult to detect. FISH with 2 probes was performed with suppression of repeated DNA by human $\mathrm{C}_{\mathrm{o}} \mathrm{t} 20 \mathrm{DNA}$ as previously described [Yudkin et al., 2014].

\section{PCR Analysis}

DNA for PCR was purified from whole blood using the Wizard Genomic DNA Purification Kit (Promega). PCR analysis was performed using Q5 High-Fidelity DNA polymerase (New England Biolabs). FMR2 genotyping was performed using 603/598 primers as previously described [Knight et al., 1993]. DNA was hydrolyzed with methyl-sensitive restrictase AspLEI (SibEnzyme) and amplified with primers for FMR1 promoter (F: $5^{\prime}$-CCCGCCCTCCACCAAGCCCGC-3' R: $5^{\prime}$-CTCCGTCACCGCCGCCGCCCG-3') for methylation analysis as formerly described [Strelnikov et al., 2000]. The CGG repeat size in the FMR1 gene promoter was determined using repeat flanking primers as described [Lokanga et al., 2013].

\section{Results}

\section{Clinical and Psychological Research Results}

A family with 3 members, a 44-year-old woman (CPG10 in the IMCB SB RAS database) and 2 sons, 25 and 20 years old (CPG9 and CPG11; Fig. 1), were examined. Both sons have mild ID and were not diagnosed earlier. The third son of CPG10 also had ID and committed suicide.

The psychological study of CPG11 showed a total underdevelopment of higher mental functions. Brain volumes in short-term memory and his memorizing speed were reduced. Memory processes were inert and exhausted. His reproduction curve was 46887 ; resistance mnemonic traces were below normal, retention was -6 . He 
Fig. 2. a Localization of the FMR1 (red) and GPR50 (green) genes in patient CPG11. Inset DAPI-stained X chromosome; the arrow indicates the fragile site FRAXA. b, c Localization of FMR1 (red) and GPR50 (green) genes in patient CPG10. Inset (b) DAPI-stained X chromosomes; the arrow indicates the fragile site FRAXA. A metaphase with $3 \mathrm{X}$ chromosomes (c) is shown. d-f Localization of X chromosome painting probe in patient CPG10. Metaphases with $3 \mathrm{X}$ chromosomes (green) (d), 2 normal X chromosomes (green) and $2 \mathrm{X}$-derived marker chromosomes (mar, green) (e), and $5 \mathrm{X}$ chromosomes (red) (f) are shown.
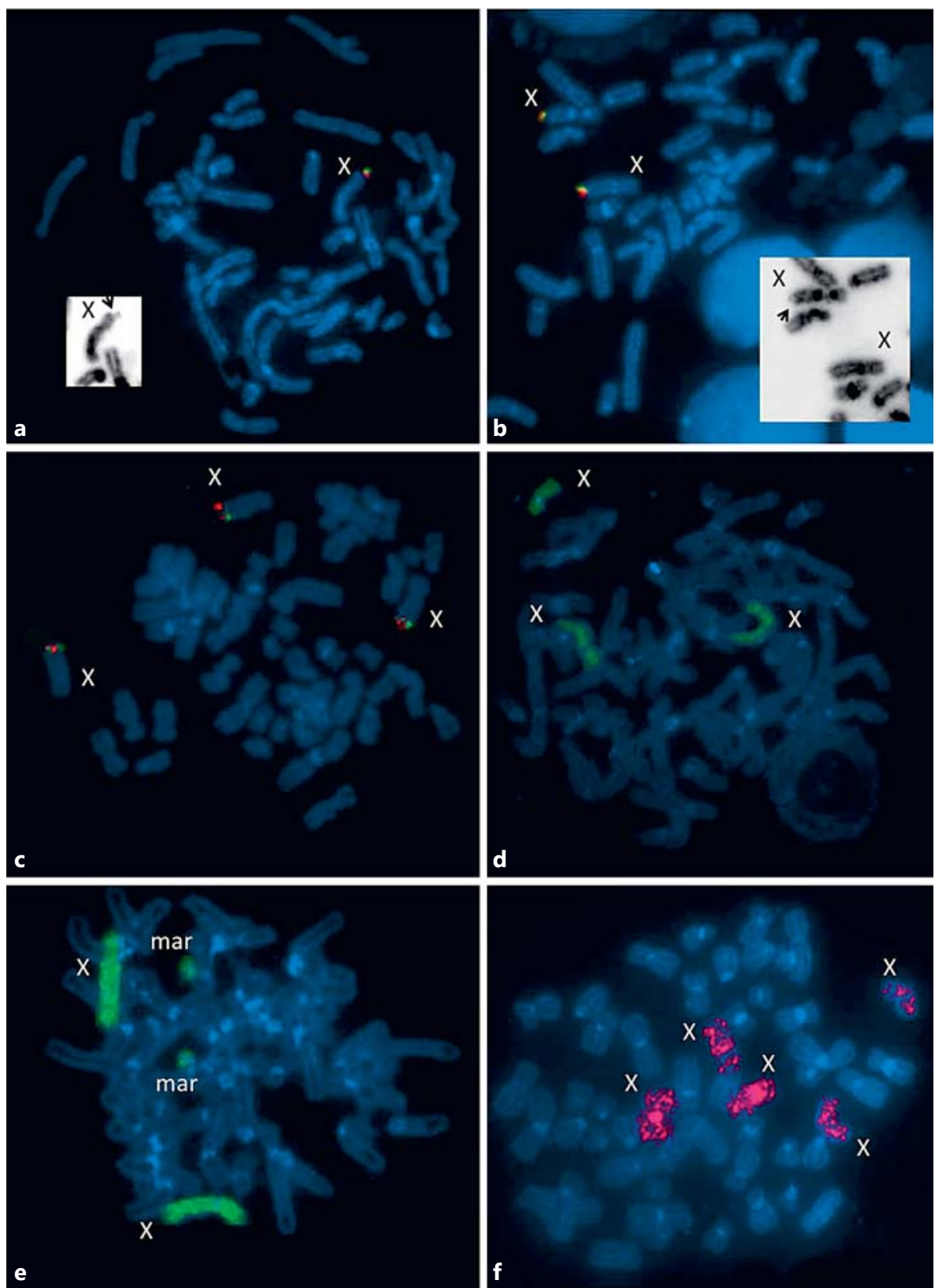

understands instructions, but does not remember them for a long time. He needs help organizing, and his tempo is slow.

He has poorly developed social orientation. His Wechsler Adult Intelligence Scale score was: verbal IQ 58, nonverbal IQ - 82, and common IQ - 67. Direct study of his emotional/personal features showed instability, diffidence, and anxiety about the invincibility of the existing problems.
The clinical psychologist diagnosed F70.0 mild ID (CPG11); the neurologist detected an encephalopathy of vascular genesis, intracranial hypertension syndrome, episodic nocturnal enuresis, and dyslalia (CPG11).

CPG9 is a patient in a psychiatric hospital, and the results of his clinical and psychological research are not available due to the clinical rules. His blood samples are available from a clinical laboratory according to ethical standards. 


\section{MRI Study}

The MRI study of CPG10 revealed solitary small nonspecific (perivascular dyscirculatory) foci in the subcortical regions in the brain. A small cyst in the left parahippocampal gyrus was found. The MRI study of CPG11 did not reveal organic brain pathology. A small asymmetry of the lateral ventricles was detected.

\section{FMR1 Allele Determination}

Repeat size was determined by amplification followed by agarose gel electrophoresis. The sample from CPG11 did not produce a PCR product. The sample from CPG9 produced a product that was the size of approximately 30 repeats, which is a normal allele, and the sample from CPG10 produced 2 products that were the size of approximately 20/160 repeats and was found to be heterozygous for the normal/permutated FMR1 allele.

The methylation analysis showed that the FMR1 promoter was methylated in CPG11 and unmethylated in CPG9. These results suggest that the absence of a repeat PCR product in CPG11 is the result of a completely mutated expanded repeat. To determine if this was the cause, we performed a molecular cytogenetic analysis with FISH using FMR1 and GPR50 probes (Fig. 2a, b). An analysis of 51 metaphases from CPG9 showed no fragile site FRAXA on the X chromosome. An analysis of 100 metaphases from CPG11 showed the presence of FRAXA in $14 \%$ of the X chromosomes (Fig. $2 \mathrm{a}$ ) as well as in $5 \%$ of the $\mathrm{X}$ chromosomes from CPG10 after the analysis of 124 metaphases (Fig. 2b).

\section{FMR2 Allele Determination}

The fragile site FRAXA is located very close to the $\mathrm{X}$ chromosome telomere and cannot be differentiated cytogenetically from the fragile site FRAXE, which is connected with repeat expansion in the FMR2 gene and is located very close to FRAXA [Knight et al., 1993]. To exclude FRAXE chromosome fragility, we determined the size of GCC repeats and found that all patients are carriers of the normal allele of FMR2 of approximately 15 triplets in length.

\section{Karyotype Analysis}

During FRAXA analysis using FISH, we found some metaphases that do not contain 2 but 3 signals from both probes in CPG10 (Fig. 2c). To analyze the number of $\mathrm{X}$ chromosomes in patients, we performed FISH with an $\mathrm{X}$ chromosome painting probe. CPG9 and CPG11 have a 46,XY karyotype. CPG10 had different rearrangements in the karyotype (Fig. 2d-f). Analysis of 369 metaphases

Female with X Abnormalities and Sons with ID revealed that $5.1 \%$ had an $\mathrm{X}$ chromosome number variation and moreover $\mathrm{X}$-derived marker chromosomes. The majority of abnormal metaphases (3.3\%) was 47,XXX (Fig. 2d), 1.6\% metaphases were 48,XX, +2der(X) (Fig. 2e), and $0.2 \%$ were 50 ,XXXXX (Fig. 2f).

\section{Discussion}

Differential diagnostics of ID is an area of great interest for modern psychiatry and molecular genetics. The involvement of hundreds of genes in the development of different forms of ID makes diagnosis complicated. Here, we report a family burdened with ID and suicide. The mother has a premutation in the FMR1 gene that is characterized by 160 CGG triplets. We describe the presence of the fragile site FRAXA in 5\% of the X chromosomes in her karyotype. Previously, FRAXA had been detected in fully mutated FMR1 only; now we can say that large premutations lead to chromosome fragility. In addition, the mother had supernumerary X chromosomes, up to pentaploidy, and X-derived marker chromosomes. Usually, supernumerary X chromosomes do not have any effect on carriers [Griffiths et al., 1999], but in this case, it must be taken into account because the mother has 1 abnormal $\mathrm{X}$ chromosome with FRAXA and children with ID and suicide. Both living sons have normal chromosome numbers and different types of ID. One of them is an FXS patient with a methylated FMR1 promoter and the FRAXA fragile site on the X chromosome. The second son has a normal FMR1 gene and his ID is not FXS based; it is of unclear nature and may be connected with any genetic or environmental cause.

In this study, we suggest the symptoms described here are due to fragile $\mathrm{X}$-associated disorders with either an additional genetic, environmental, or social background for ID.

\section{Acknowledgments}

We thank Professor Malcolm A. Ferguson-Smith at the University of Cambridge for providing the $\mathrm{X}$ chromosome painting probe. This study is supported by the Russian Science Foundation (grant 15-15-10001).

\section{Statement of Ethics}

This study was designed strictly in accordance with international standards, which include the awareness of the subject, his or her consent to participating in the study in its entirety, and guar- 
antees of confidentiality. All of the studies conformed to ethical standards developed in accordance with the Helsinki Declaration of the World Medical Association as amended in 2000. In addition, the studies were supervised by the Institutional Review Board.

\section{Disclosure Statement}

The authors declare no conflicts of interests.

\section{References}

D’Hulst C, Kooy RF: Fragile X syndrome: from molecular genetics to therapy. J Med Genet 46:577-584 (2009).

Dobkin C, Radu G, Ding XH, Brown WT, Nolin SL: Fragile X prenatal analyses show full mutation females at high risk for mosaic Turner syndrome: fragile $\mathrm{X}$ leads to chromosome loss. Am J Med Genet Part A 149A:2152-2157 (2009).

Fryns JP, Kleczkowska A, Petit P, van den Berghe $\mathrm{H}$ : X-chromosome polysomy in the female: personal experience and review of the literature. Clin Genet 23:341-349 (1983).

Griffiths AJF, Miller JH, Suzuki DT, Lewontin RC, Gelbart WM: An Introduction to Genetic Analysis, ed 7 (WH Freeman \& Co Press, New York 1999).
Knight SJ, Flannery AV, Hirst MC, Campbell L, Christodoulou Z, et al: Trinucleotide repeat amplification and hypermethylation of a $\mathrm{CpG}$ island in FRAXE mental retardation. Cell 74: 127-134 (1993).

Lee NR, Wallace GL, Adeyemi EI, Lopez KC, Blumenthal JD, et al: Dosage effects of $\mathrm{X}$ and $\mathrm{Y}$ chromosomes on language and social functioning in children with supernumerary sex chromosome aneuploidies: implications for idiopathic language impairment and autism spectrum disorders. J Child Psychol Psychiatry 53:1072-1081 (2012).

Lokanga RA, Entezam A, Kumari D, Yudkin D, Qin M, et al: Somatic expansion in mouse and human carriers of fragile $\mathrm{X}$ premutation alleles. Hum Genet 34:157-166 (2013).
Strelnikov V, Nemtsova M, Blinnikova O, Chesnokava G, Kuleshov N, Zaletaev D: Modern methods for DNA-diagnostics of Martin-Bell syndrome. Pediatrics (Rus) 4:21-25 (2000).

Telenius H, Pelmear AH, Tunnacliffe A, Carter NP, Behmel A, et al: Cytogenetic analysis by chromosome painting using DOP-PCR amplified flow-sorted chromosomes. Genes Chromosomes Cancer 4:257-263 (1992).

Vissers LE, Gilissen C, Veltman JA: Genetic studies in intellectual disability and related disorders. Nat Rev Genet 17:9-18 (2016).

Yudkin D, Hayward BE, Aladjem MI, Kumari D, Usdin K: Chromosome fragility and the abnormal replication of the FMR1 locus in fragile X syndrome. Hum Mol Genet 23:29402952 (2014) 